Pacific

Journal of

Mathematics

SIMPLE CONNECTIVITY OF THE MARKOV PARTITION SPACE

L. BADOIAN AND J.B. WAGONER

Volume 193 No. 1

March 2000 


\title{
SIMPLE CONNECTIVITY OF THE MARKOV PARTITION SPACE
}

\author{
L. BADOIAN AND J.B. WAGONER
}

In Wagoner, 1987 the simplicial complex $P_{A}$ of Markov partitions was introduced as a tool for studying the group of automorphisms of a subshift of finite type $\left(X_{A}, \sigma_{A}\right)$ built from a zero-one transition matrix $A$. Triangles in $P_{A}$ led to the matrix Triangle Identities in Wagoner, Pac. Journal, 1990 which have been used in Wagoner, 1990, 1990, 1990, 1992, Kim, Roush \& Wagoner, 1992, and the Williams Conjecture counterexample paper Kim \& Roush, to appear.

A key fact about $P_{A}$ is that it is contractible. See Wagoner, 1987. The purpose of this note is to correct the proof on pp. 99-100 in Wagoner, 1987 that $P_{A}$ is simply connected and in the process to improve the bound in Proposition 2.13 of Wagoner, 1987.

Proposition. A closed path in $P_{A}$ with $L$ edges can be spanned by a (possibly singular) triangulated 2 -disc in $P_{A}$ having at most $8 L^{2}+L$ triangles.

The difficulty with the proof of (2.13) in [W1] occurs in the diagram of Step 2 on p. 99, because it may not be the case that $V_{i-1} \cap V_{i+1}$ is a Markov partition.

To correct this, it is better to change to a more straightforward notation and let $U \longrightarrow V$ rather than $V \longrightarrow U$ mean $V<U<\sigma_{A}(V) \cap V$. Recall from $[\mathbf{W} 1]$ that $U \underset{+}{\longrightarrow} V$ means $U<V<U \cap \sigma_{A}^{-1}(U)$. Then Definition 2.10 of $[\mathbf{W} \mathbf{1}]$ becomes

$$
U \rightarrow V \quad \text { iff } \quad U \underset{+}{\longrightarrow} U \cap V \underset{-}{\longrightarrow} V
$$

In particular, now $U \longrightarrow V$ implies that $U \rightarrow V$ in $P_{A}$ but with extra information, whereas in [W1] the notation $V \rightarrow U$ implied $U \rightarrow V$, which is somewhat contrary. Here are some properties of the arrows $U \underset{+}{\longrightarrow} V$ and $U \longrightarrow V$. See $[\mathbf{W} \mathbf{1}]$.

1) If $U \underset{+}{\longrightarrow} V$ and $U$ is a Markov partition, then $V$ is a Markov partition. If $U \longrightarrow V$ and $V$ is a Markov partition, then $U$ is a Markov partition. 
2) If $U \rightarrow V$ and $U$ and $V$ are Markov partitions, then $U \cap V$ is a Markov partition.

3) If $U \underset{+}{\longrightarrow} V, W$ and $U$ is a Markov partition, then $U \underset{+}{\longrightarrow} \cap W$ and $V \cap W$ is a Markov partition. If $U, V \rightarrow W$ and $W$ is a Markov partition, then $U \cap V \rightarrow W$ and $U \cap V$ is a Markov partition.

4) If $U \underset{-}{\longrightarrow} \underset{+}{\longrightarrow} V$ and $U, X$, and $V$ are Markov partitions, then $U \underset{+}{\longrightarrow}$ $U \cap V \longrightarrow V$ and $U \cap V$ is a Markov partition.

For completeness, we recall Definition 2.11 of [W1] giving the simplicial structure on $P_{A}$. Namely, an $n$-simplex of $P_{A}$ is an ordered $(n+1)$-tuple $\left\langle V_{0}, V_{1}, \ldots, V_{n}\right\rangle$ such that $V_{i} \rightarrow V_{j}$ whenever $i \leq j$.

The next step is to replace the diagram in Step 2 on p. 99 with the following diagram

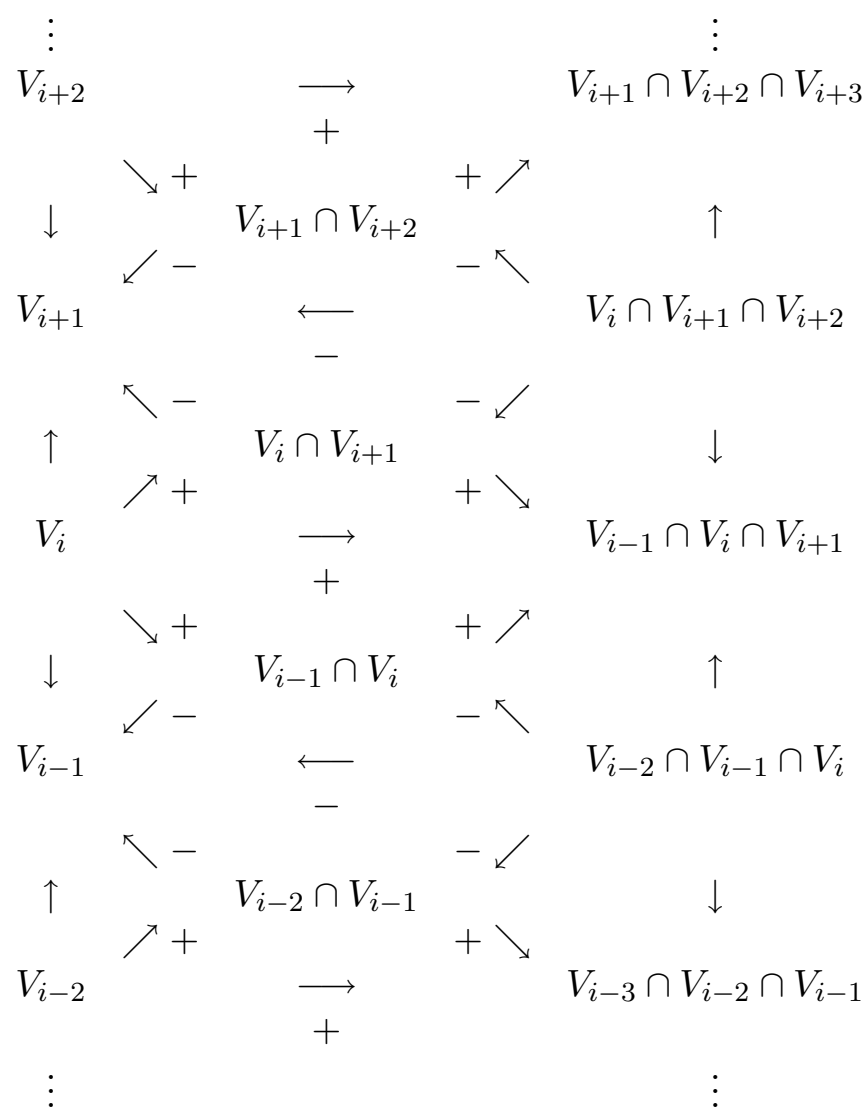

We can now deform a closed path of length L to a constant path as follows: Step 1 on p. 99 deforms the closed path of length $\mathrm{L}$ to an alternating 
closed path of length $2 \mathrm{~L}$ with vertices $V_{0}, V_{1}, \ldots, V_{2 L}$. The number of triangles in this deformation is at most $\mathrm{L}$. Then the above diagram deforms the alternating closed path of length $2 \mathrm{~L}$ on the left to an alternating closed path of length $2 \mathrm{~L}$ on the right with vertices of the form $V_{i-1} \cap V_{i} \cap V_{i+1}$. Repeating the deformation in the diagram L-1 more times produces an alternating closed path of length $2 \mathrm{~L}$ with vertices of the form

$$
V_{i-L} \cap V_{i-L+1} \cap \ldots \cap V_{i-1} \cap V_{i} \cap V_{i+1} \ldots \cap V_{i+L-1} \cap V_{i+L} .
$$

Thus all the vertices in this path are equal to

$$
V_{0} \cap V_{1} \cap \ldots \cap V_{2 L} .
$$

The total number of triangles in this deformation is at most $8 L^{2}+L$.

Remark. The argument in $[\mathbf{W} 1]$ that $H_{n}\left(P_{A}\right)=0$ for $n \geq 2$ avoids the $V_{i-1} \cap V_{i+1}$ type difficulty, because all intersections of Markov partitions encountered in the proof are Markov partitions as a consequence of properties (1) through (4) above. There is a typographical change on p. 102, 1.10: $V_{p_{s}} \cap V_{p_{s}}$ should read $V_{p_{s}} \cap V_{q_{s}}$.

\section{References}

[KR] K.H. Kim and F.W. Roush, Williams' Conjecture is false for irreducible subshifts, 1997, preprint from Alabama State University at Montgomery, to appear in Annals of Mathematics.

[KRW] K.H. Kim, F.W. Roush and J.B. Wagoner, Automorphisms of the dimension group and gyration numbers, Jour. Amer. Math. Soc., 5(1) (1992), 191-212.

[W1] J.B. Wagoner, Markov partitions and $K_{2}$, Pub. Math. IHES, 65 (1987), 91-129.

[W2] , Triangle identities and symmetries of a subshift of finite type, Pac. Jour. Math., 144 (1990), 181-205.

[W3] _ Eventual finite order generation for the kernel of the dimension group representation, Trans. Amer. Math. Soc., 317(1) (1990), 331-350.

[W4] Higher dimensional shift equivalence and strong shift equivalence are the same over the integers, Proc. Amer. Math. Soc., 109(2) (1990), 527-536.

[W5] _ Classification of subshifts of finite type revisited, Contemporary Mathematics, 135 (1992), 423-444.

Received May 5, 1998. The authors were partially supported by NSF Grant \# DMS 9322498 .

Department of Mathematics

UNIVERSiTy OF CALIFORNIA

BERKELEY, CA 94720

E-mail address: badoian@math.berkeley.edu

Department of Mathematics

UNIVERSITY OF CALIFORNIA

BERKELEY, CA 94720 
E-mail address: wagoner@math.berkeley.edu 